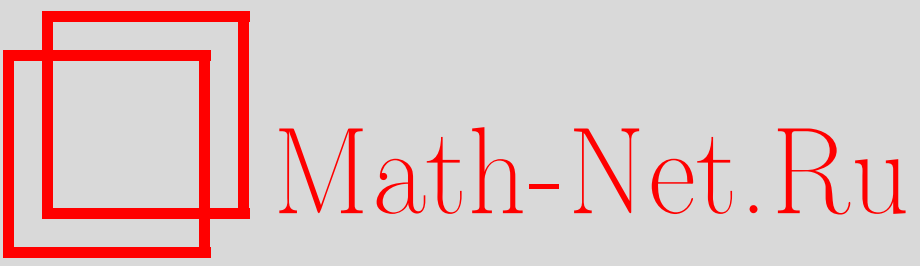

Е. С. Голод, Некоммутативные полные пересечения и гомологии комплекса Шафаревича, УМН, 1997, том 52, выпуск 4, 201-202

DOI: https://doi.org/10.4213/rm869

Использование Общероссийского математического портала Math-Net.Ru подразумевает, что вы прочитали и согласны с пользовательским соглашением

http://www.mathnet.ru/rus/agreement

Параметры загрузки:

IP: 3.95 .254 .165

26 апреля 2023 г., 12:01:03 


\title{
НЕКОММУТАТИВНЫЕ ПОЛНЫЕ ПЕРЕСЕЧЕНИЯ И ГОМОЛОГИИ КОМПЛЕКСА ШАФАРЕВИЧА
}

\author{
Е. С. Голод
}

Аналогом хорошо известного в коммутативной алгебре понятия регулярной последовательности в некоммутативной ситуации служит понятие сильно свободного, или инертного, множества элементов, которое определяется рядом эквивалентных (при некоторых ограничениях) условий [2], из которых наиболее удобно для далшнейшего следующее. Пусть $R$ - ассоциативная алгебра над коммутативным кольцом $k, x=\left\{x_{\alpha}\right\}$ - семейство элементов из $R$ и $\operatorname{Sh}(x, R)$ - соответствующий комплекс Шаффаревича [1], [4]. Семейство $\left\{x_{\alpha}\right\}$ называется силшно свободным, если $H_{i}(\operatorname{Sh}(x, R))=0$ при $i>0$. Алгебры, представимые как факторалгебры свободной ассоциативной алгебры по идеалу, порожденному сильно свободным множеством, естественно рассматривать как некоммутативные аналоги полных пересечений в коммутативной алгебре. Известная теорема Тейта-Ассмуса [3], [5] характеризует коммутативные (локальные или градуированные) полные пересечения тем свойством, что алгебра гомологий их комплекса Козюля является внешней алгеброй над пространством одномерных гомологий. В настоящей статье анонсируется некоммутативный аналог (обобщенного варианта) этого результат (теорема 1 ). Теорема 2 , не имеющая аналога в коммутативном случае, показывает, что алгебра гомологий комплекса Шафаревича для свободной алгебры обладает весьма специальными (если принять во внимание теорему 1) свойствами.

Пусть $(A, \partial)$ - дифференциалнная градуированная (DG) алгебра (с единицей) над коммутативньм кольцом $k$ с дифференциалом $\partial$ степени -1 (градуировка и дифференциал могут быть тривиальными), $U$-градуированньй $k$-модуль и $\varphi: U \rightarrow A$-однородный степени $-1 k$-линейньй гомоморфизм, для которого $\operatorname{Im}(\varphi) \subseteq \operatorname{Ker}(\partial)$. Через $S=\operatorname{Sh}(U, \varphi, A)$ будем обозначать $k$-алгебру, представляющую собой свободное произведение $A * T(U)$ над $k$ алгебры $A$ и тензорной алгебры $T(U)$. Градуировка на $A$ и $U$ естественно определяют градуировку на $S$, и на градуированной алгебре $S$ имеется однозначно определенньй дифференциал $d$, совпадающий с $\partial$ на $A$ и с $\varphi$ на $U$, которьй превращает $S$ в DG-алгебру над $k$. Легко показать, что когда $U-$ проективный $k$-модуль, структура DG-алгебры на $S$ с точностью до изоморфизма зависит только от индуцированного $\varphi$ отображения $U \rightarrow H_{*}(A)$. Конструкция комплекса $\operatorname{Sh}(U, \varphi, A)$ является некоторым обобщением конструкции комплекса Шафаревича и одновременно некоммутативным аналогом конструкции резольвенты Тейта [5].

Если DG-алгебра $(A, \partial)$ имеет тривиальньй дифференциал $\partial$, то, заменяя градуировку на $A$ тривиальнй и наделяя модуль $U$ соответственно градуировкой, сосредоточенной в степени +1 , мы получим на алгебре $S$ градуировку, задаваемую прямьм разложением

$$
S=A \oplus A \otimes U \otimes A \oplus A \otimes U \otimes A \otimes U \otimes A \oplus \cdots
$$

(тензорное произведение над $k$ ), которую будем назьвать $U$-градуировкой. Модули гомологий комплекса $(S, d)$ относительно $U$-градуировки будем обозначать через $H_{(p)}(S)$.

Далее мы будем рассматривать только случай, когда $U$ - свободный $k$-модуль с некоторым базисом $u=\left\{u_{\alpha}\right\}$; тогда $x=\left\{x_{\alpha}\right\}$, где $x_{\alpha}=\varphi\left(u_{\alpha}\right),-$ семейство однородных циклов из $A$, заданием которого определяется отображение $\varphi$, и вместо $\operatorname{Sh}(U, \varphi, A)$ будем использовать обозначение $\operatorname{Sh}(u \mid x, A)$, уточняющее обозначение $\operatorname{Sh}(x, A)$, применявшееся в [4].

Пусть теперь $A$ - градуированная алгебра над полем $k, x=\left\{x_{\alpha}\right\}$ - некоторое семейство однородных элементов из $A, I=\left\langle\left\{x_{\alpha}\right\}\right\rangle$ - порожденный им идеал в $A$ и $y=\left\{y_{\beta}\right\}$ - некоторое семейство однородных элементов из $I$. Рассмотрим какое-либо представление элементов $y_{\beta}$ в виде

$$
y_{\beta}=\sum_{i} a_{i, \beta} x_{\alpha_{i}} b_{i, \beta}
$$

где $a_{i, \beta}, b_{i, \beta} \in A$ однородные. Пусть $J$ - идеал в $A$, порожденньй семейством $y$, и $B=A / J$. Канонический образ элемента $a \in A$ в $B$ обозначаем через $\bar{a}$. B DG-алгебре $\operatorname{Sh}(u \mid \bar{x}, B)$ рассмотрим семейство $z=\left\{z_{\beta}\right\}$ однородных циклов $z_{\beta}=\sum_{i}(-1)^{\operatorname{deg} a_{i, \beta}} \bar{a}_{i, \beta} u_{\alpha_{i}} \bar{b}_{i, \beta}$. Легко видеть,

Работа вьполнена при частичной поддержке Российского фонда фундаментальных исследований (грант № 96-01-00149). 
что класс гомологий цикла $z_{\beta}$ не зависит от выбора представления элемента $y_{\beta}$ в виде (1). Пусть $V$ - градуированное векторное пространство над $k$ с базисом $v=\left\{v_{\beta}\right\}$, где $\operatorname{deg} v_{\beta}=\operatorname{deg} y_{\beta}+1$. На алгебрах $H_{*}(\operatorname{Sh}(u \mid x, A)) * T(V)$ и $H_{*}(\operatorname{Sh}(u \mid \bar{x}, B))$ помимо уже имеющихся градуировок рассмотрим " $U$-градуировки", задаваемые разложениями

$$
H_{*}(\operatorname{Sh}(u \mid x, A))=\bigoplus_{p} H_{(p)}(\operatorname{Sh}(u \mid x, A)), \quad H_{*}(\operatorname{Sh}(u \mid \bar{x}, B))=\bigoplus_{p} H_{(p)}(\operatorname{Sh}(u \mid \bar{x}, B))
$$

и приписыванием элементам из $V$ степени +1 . Под степенью элемента будем понимать степень относительно исходных градуировок, а степень относительно $U$-градуировок будем назьвать размерностью. Рассмотрим однородный относительно обеих градуировок гомоморфизм $k$-алгебр $\pi_{*}: H_{*}(\operatorname{Sh}(u \mid x, A)) * T(V) \rightarrow H_{*}(\operatorname{Sh}(u \mid \bar{x}, B))$, индуцированный естественным отображением $H_{*}(\operatorname{Sh}(u \mid x, A)) \rightarrow H_{*}(\operatorname{Sh}(u \mid \bar{x}, B))$ и отображением $T(V) \rightarrow H_{*}(\operatorname{Sh}(u \mid \bar{x}, B))$, при котором каждый $v_{\beta}$ отображается в класс гомологий цикла $z_{\beta}$.

Теорема 1. Рассмотрим следующие условия:

1) $y=\left\{y_{\beta}\right\}-$ сильно свободное множсество в $A$;

2) отображение $\pi_{*}-$ изоморфизм;

3) естественное отображсение $H_{*}(\operatorname{Sh}(u \mid x, A)) \rightarrow H_{*}(\operatorname{Sh}(t \mid z, \operatorname{Sh}(u \mid \bar{x}, B)))$ является изоморфизмом;

4) отображение $h_{*}$ ингективно на пространстве одномерных әлементов и сюрдективно на пространстве двумерных әлементов.

Имеют место импликации: 1$) \Rightarrow 2) \Rightarrow 3) \Rightarrow 4)$. Если элементы $x_{\alpha}$ имеют положительную степень, то все четьре условия әквивалентны.

СледСтвиЕ. Пусть $A=k\left\langle\left\{x_{\alpha}\right\}\right\rangle$ - свободная ассочиативная алгебра над полем $k$, свободным порождающим которой приписаны произвольные положительнье степени, и B - факторалгебра алгебры А по некоторому однородному идеалу. Тогда следующие условия әквивалентны:

1) $B$ - некоммутативное полное пересечение;

2) $H_{*}(\operatorname{Sh}(u \mid \bar{x}, B))$ - тензорная алгебра над пространством одномерных гомологий $H_{(1)}(\operatorname{Sh}(u \mid \bar{x}, B))$;

3) пусть $z=\left\{z_{\beta}\right\}$ - семейство одномерньх циклов в $\operatorname{Sh}(u \mid \bar{x}, B)$, классь гомологий которых $h_{\beta}$ образуют базис пространства $H_{(1)}(\operatorname{Sh}(u \mid \bar{x}, B)) u T=\operatorname{Sh}(t \mid z, \operatorname{Sh}(u \mid \bar{x}, B))$; тогда $H_{(p)}(T)=0$ при $p>0$;

4) $H_{(2)}(\operatorname{Sh}(u \mid \bar{x}, B))=H_{(1)}(\operatorname{Sh}(u \mid \bar{x}, B))^{2}$.

Эквивалентность условий 2) и 3 ) из теоремы 1 обобщается следующим утверждением.

ПреДЛОЖЕНИЕ. Пусть $A-\mathrm{DG}-а л г е б р а, z=\left\{z_{\beta}\right\}-$ семейство однородных ииклов положительной степени в $A$ и $N$ - идеал в $H_{*}(A)$, порожденный классами гомологий $h_{\beta}$ ииклов $z_{\beta}$. Естественное отображение $H_{*}(A) / N \rightarrow H_{*}(\operatorname{Sh}(t \mid z, A))$ является изоморфизмом в том и только в том случае, когда $\left\{h_{\beta}\right\}$ - сильно свободное множество в $H_{*}(A)$.

Теорема 2. Пусть $A-$ свободная ассочиативная алгебра над полем $k$ u $f=\left\{f_{\alpha}\right\}-$ произвольное семейство әлементов из $A$. Тогда $H_{*}(u \mid f, A)$ порожсдается как $k$-алгебра нульмерными и одномерными әлементами.

\section{СПИСОК ЛИТЕРАТУРЫ}

[1] Голод Е. С., Шафаревич И.Р. // Изв. АН СССР. Сер. матем. 1964. Т. 28. № 2. C. 261-272. [2] Anick D. // J. Algebra. 1982. V. 78. P. 120-140. [3] Assmus E. F., Jr. // Illinois J. Math. 1959. V. 3. № 2. P. 187-199. [4] Golod E. S. // Lect. Notes in Math. 1988. V. 1352. P. 88-95. [5] Tate J. // Illinois J. Math. 1957. V. 1. № 1. P. 14-27. 